\title{
Perturbation of a Nonlinear Elliptic Biological Interacting Model
}

\author{
Kami Lizarraga, Joon Hyuk Kang, and Jungho Lee
}

Communicated by Y. Charles Li, received September 7, 2005.

\begin{abstract}
In this paper, we investigate the effects of perturbations on the coexistence state of the general competition model for two species. Previous work by Kang, Lee, and Oh (see [10]) established sufficient conditions for the uniqueness of the positive solution to the following general elliptic system for two competing species of animals:

$$
\left\{\begin{array}{c}
\Delta u+u g(u, v)=0 \\
\Delta v+v h(u, v)=0 \\
\left.u\right|_{\partial \Omega}=\left.v\right|_{\partial \Omega}=0
\end{array} \quad \text { in } \Omega,\right.
$$

That is, they proved that under certain conditions, two species can coexist and that the coexistence state is unique at fixed rates. In this paper, we extend their uniqueness results by perturbing functions $g$ and $h$ of the above model, and applying super-sub solutions, maximum principles and spectrum estimates. Our arguments also rely on some detailed properties for the solution of logistic equations. By applying these techniques, we obtain sufficient conditions for the existence and uniqueness of a time independent coexistence state for the perturbed general competition model.
\end{abstract}

\section{Introduction}

One of the prominent subjects of study and analysis in mathematical biology concerns the competition of two or more species of animals in the same environment. Especially pertinent areas of investigation include the conditions under which the species can coexist, as well as the conditions under which any one of the species becomes extinct, that is, one of the species is excluded by the others. In this paper, we focus on the general competition model to better understand the competitive interactions between two species. Specifically, we investigate the conditions needed for the coexistence of two species when the factors affecting them are perturbed.

1991 Mathematics Subject Classification. 35A05, 35A07.

Key words and phrases. Lotka Volterra competition model, coexistence state.

This research was supported by Andrews University Undergraduate Research Assistant Fund 2004, 2005. 


\section{Literature Review}

Within the academia of mathematical biology, extensive academic work has been devoted to investigation of the simple competition model, commonly known as the Lotka-Volterra competition model. This system describes the competitive interaction of two species residing in the same environment in the following manner:

$$
\left\{\begin{array}{c}
u_{t}(x, t)=\Delta u(x, t)+u(x, t)(a-b u(x, t)-c v(x, t)) \quad \text { in } \Omega \times R^{+}, \\
v_{t}(x, t)=\Delta v(x, t)+v(x, t)(d-f v(x, t)-e u(x, t)) \\
\left.u(x, t)\right|_{\partial \Omega}=\left.v(x, t)\right|_{\partial \Omega}=0,
\end{array}\right.
$$

where $\Omega$ is a bounded domain in $R^{n}$. Here, $u(x, t)$ and $v(x, t)$ designate the population densities for the two competing species. The positive constant coefficients in this system represent growth $\operatorname{rates}(a$ and $d)$, self-limitation $\operatorname{rates}(b$ and $f)$ and competition rates $(c$ and $e)$. Furthermore, we assume that both species are not residing on the boundary of $\Omega$.

The mathematical community has already established several results for the existence, uniqueness and stability of the positive steady state solution to (1), see $[\mathbf{1}],[\mathbf{2}],[\mathbf{3}],[\mathbf{4}],[\mathbf{6}],[\mathbf{7}]$. The positive steady state solution is simply the positive solution to the time-independent system

$$
\left\{\begin{array}{c}
\Delta u(x)+u(x)(a-b u(x)-c v(x))=0 \\
\Delta v(x)+v(x)(d-f v(x)-e u(x))=0 \\
\left.u(x)\right|_{\partial \Omega}=\left.v(x)\right|_{\partial \Omega}=0 .
\end{array} \text { in } \Omega,\right.
$$

One of the initial important results for the time-independent Lotka-Volterra model was obtained by Cosner and Lazer. In 1984, they published the following sufficient conditions for the existence and uniqueness of a positive steady state solution to (2):

THEOREM 2.1. (in [4])

Suppose

(A) $a>\lambda_{1}+\frac{c d}{f}, d>\lambda_{1}+\frac{a e}{b}$, where $\lambda_{1}$ is the smallest eigenvalue of $-\Delta$ with homogeneous boundary conditions,

(B) $4 b f>\frac{f c^{2}}{b} \sup _{x \in \Omega}\left[\frac{\omega_{a}(x)}{\omega_{d-\frac{a e}{b}(x)}}\right]+2 c e+\frac{b e^{2}}{f} \sup _{x \in \Omega}\left[\frac{\omega_{d}(x)}{\omega_{a-\frac{c d}{f}}(x)}\right]$, where $\omega_{M}(x)$ for $M>0$ is the unique positive solution to the logistic equation as mentioned in the next section.

Then (2) has a unique positive solution.

Cosner and Lazer's theorem implies that if the self-reproduction and selflimitation rates are relatively large, and the competition rates are relatively small, then there is a unique positive steady state solution to (2). In other words, the two species will coexist indefinitely at unique population densities.

In 1989, Cantrell and Cosner extended these results by proving that the reproduction and self limitation rates may vary within bounds without losing the uniqueness result, given certain conditions. Biologically, Cantrell and Cosner's theorem suggests that two species can relax ecologically and maintain a coexistence state. Their primary result is given below: 
THEOREM 2.2. (in $[\mathbf{3}])$

If $a=d>\lambda_{1}, b=f=1$, and $0<c, e<1$, then there is a neighborhood $V$ of $(a, a)$ such that if $\left(a_{0}, d_{0}\right) \in V$, then (2) with $(a, d)=\left(a_{0}, d_{0}\right)$ has a unique positive solution.

In Theorem 2.2, the condition $0<c, e<1$ biologically implies that the competition rates of both species must be relatively small. This condition plays an important role in the proof of Cantrell and Cosner's theorem by implying the invertibility of the Frechet derivative (linearization) of (2) at a fixed reproduction rate $(a, a)$.

The work of Lazer, Cosner, and Cantrell provides insight into the competitive interactions of two species operating under the conditions described in the LotkaVolterra model. However, their results are somewhat limited by a few key assumptions. In the Lotka-Volterra model that they studied, the rate of change of densities largely depends on constant rates of reproduction, self-limitation, and competition. The model also assumes a linear relationship of the terms affecting the rate of change for both population densities.

However, in reality, the rates of change of population densities may vary in a more complicated and irregular manner than can be described by the simple competition model. Therefore, in the last decade, significant research has been focused on the existence and uniqueness of the positive steady state solution of the general competition model for two species,

$$
\left\{\begin{array}{r}
u_{t}(x, t)=\Delta u(x, t)+u(x, t) g(u(x, t), v(x, t)) \\
v_{t}(x, t)=\Delta v(x, t)+v(x, t) h(u(x, t), v(x, t)) \\
\left.u(x, t)\right|_{\partial \Omega}=\left.v(x, t)\right|_{\partial \Omega}=0
\end{array}\right.
$$

or, equivalently, the positive solution to

$$
\left\{\begin{array}{c}
\Delta u(x)+u(x) g(u(x), v(x))=0 \\
\Delta v(x)+v(x) h(u(x), v(x))=0 \\
\left.u\right|_{\partial \Omega}=\left.v\right|_{\partial \Omega}=0
\end{array} \quad \text { in } \Omega,\right.
$$

where $g, h \in C^{1}$ designate reproduction, self-limitation and competition rates that satisfy the growth conditions $(G 1)$ and $(G 2)$ given below (see [8], [9], [10], [11], [12]).

Because of its broader applicability, the general competition model has become a more popular subject of research within the mathematical community over the past few years. In 2004, Kang, Oh, and Lee (see [10]) established the following existence, nonexistence and uniqueness result, which generalizes Theorem 2.1 obtained by Cosner and Lazer.

TheOrem 2.3. Suppose

$(G 1) g_{u}(u, v)<0, g_{v}(u, v)<0, h_{u}(u, v)<0, h_{v}(u, v)<0$,

$(G 2)$ there exist constants $c_{0}>0, c_{1}>0$ such that $g(u, 0) \leq 0$ for $u \geq c_{0}$ and $h(0, v) \leq 0$ for $v \geq c_{1}$.

(1) If $g\left(0, c_{1}\right)>\lambda_{1}$ and $h\left(c_{0}, 0\right)>\lambda_{1}$, then (3) has a coexistence state. 
(2) If $g(0,0) \leq \lambda_{1}$ or $h(0,0) \leq \lambda_{1}$, then (3) does not have any positive solution.

(3) If $g\left(0, c_{1}\right)>\lambda_{1}, h\left(c_{0}, 0\right)>\lambda_{1}$ and

$$
\begin{aligned}
4 \inf \left(-g_{u}(u, v)\right) \inf \left(-h_{v}(u, v)\right) \geq & \frac{\theta_{g(\cdot, 0)}}{\theta_{h\left(c_{0}, \cdot\right)}}\left(\sup \left(g_{v}(u, v)\right)\right)^{2}+\frac{\theta_{h(0, \cdot)}}{\theta_{g\left(\cdot, c_{1}\right)}}\left(\sup \left(h_{u}(u, v)\right)\right)^{2} \\
& +2 \sup \left(g_{v}(u, v)\right) \sup \left(h_{u}(u, v)\right),
\end{aligned}
$$

Then (3) has a unique coexistence state.

Biologically, we can interpret the conditions in Theorem 2.3 as follows. The functions $g, h, g_{u}, g_{v}, h_{u}$, and $h_{v}$ describe the manner in which members of each species $u$ and $v$ interact among themselves and with members of the other species. Hence, the conditions imply that members of each species interact strongly among themselves and weakly with members of the other species. If these conditions are fulfilled, then the two species residing within the same domain will coexist indefinitely at unique densities.

An especially significant aspect of the global uniqueness result is the stability of the positive steady state solution, which has become an important subject of mathematical study. Indeed, researchers have obtained several stability results for the Lotka-Volterra model with constant rates(see [3], [4], [7], [12]). However, the stability of the steady state solution for the general model remains open to investigation.

The research presented in this paper therefore begins the mathematical community's discussion on the stability of the steady state solution for the general competition model. In our analysis we focus on the conditions required for the maintenance of the coexistence state of (3)when bounded functions $g$ and $h$ are slightly perturbed. Mathematically, our results will generalize Theorem 2.2 developed by Cantrell and Cosner. Biologically, our conclusion implies that two species may slightly relax ecologically and yet continue to coexist at unique densities.

\section{Preliminaries}

Before entering into our primary arguments and results, we must first present a few preliminary items that we later employ throughout the proofs detailed in this paper. The following definition and lemmas are established and accepted throughout the literature on our topic.

Definition 3.1. (Super and sub solutions) Consider

$$
\left\{\begin{array}{l}
\Delta u+f(x, u)=0 \text { in } \Omega, \\
\left.u\right|_{\partial \Omega}=0
\end{array}\right.
$$

where $f \in C^{\alpha}(\bar{\Omega} \times R)$ and $\Omega$ is a bounded domain in $R^{n}$. (A) A function $\bar{u} \in C^{2, \alpha}(\bar{\Omega})$ satisfying

$$
\left\{\begin{array}{l}
\Delta \bar{u}+f(x, \bar{u}) \leq 0 \text { in } \Omega \\
\left.\bar{u}\right|_{\partial \Omega} \geq 0
\end{array}\right.
$$

is called a super solution to (4).

(B) A function $\underline{u} \in C^{2, \alpha}(\bar{\Omega})$ satisfying

$$
\left\{\begin{array}{l}
\Delta \underline{u}+f(x, \underline{u}) \geq 0 \text { in } \Omega, \\
\left.\underline{u}\right|_{\partial \Omega} \leq 0
\end{array}\right.
$$


is called a sub solution to (4).

Lemma 3.1. Let $f(x, \xi) \in C^{\alpha}(\bar{\Omega} \times R)$ and let $\bar{u}, \underline{u} \in C^{2, \alpha}(\bar{\Omega})$ be, respectively, super and sub solutions to (4) which satisfy $\underline{u}(x) \leq \bar{u}(x), x \in \bar{\Omega}$. Then (4) has a solution $u \in C^{2, \alpha}(\bar{\Omega})$ with $\underline{u}(x) \leq u(x) \leq \bar{u}(x), x \in \overline{\bar{\Omega}}$.

In our proof, we also employ accepted conclusions concerning the solutions of the following logistic equations.

LEMma 3.2. (Established in [11])

Consider

$$
\left\{\begin{array}{l}
\Delta u+u f(u)=0 \text { in } \Omega, \\
\left.u\right|_{\partial \Omega}=0, u>0
\end{array}\right.
$$

where $f$ is a decreasing $C^{1}$ function such that there exists $c_{0}>0$ such that $f(u) \leq 0$ for $u \geq c_{0}$ and $\Omega$ is a bounded domain in $R^{n}$.

If $f(0)>\lambda_{1}$, then the above equation has a unique positive solution, where $\lambda_{1}$ is the first eigenvalue of $-\Delta$ with homogeneous boundary conditions. We denote this unique positive solution as $\theta_{f}$.

The most important property of this positive solution, for the purposes of our research, is that $\theta_{f}$ is increasing as $f$ is increasing.

We specifically note that for $a>\lambda_{1}$, the unique positive solution of

$$
\left\{\begin{array}{l}
\Delta u+u(a-u)=0 \text { in } \Omega \\
\left.u\right|_{\partial \Omega}=0, u>0
\end{array}\right.
$$

is denoted by $\omega_{a} \equiv \theta_{a-x}$. Hence, $\theta_{a}$ is increasing as $a>0$ is increasing.

Having established these preliminaries, we now commence our investigation of perturbations of the general competition model.

\section{Perturbation}

We consider the model

$$
\left\{\begin{array}{c}
\Delta u+u g(u, v)=0 \\
\Delta v+v h(u, v)=0 \\
\left.u\right|_{\partial \Omega}=\left.v\right|_{\partial \Omega}=0
\end{array} \quad \text { in } \Omega,\right.
$$

Here $\Omega$ is a smooth, bounded domain in $R^{n}$. Also, two growth conditions hold:

(P1) $g, h \in C_{B}^{1}$, where $C_{B}^{1}$ is the set of all functions $f$ such that $f$ is decreasing, and all the first-order partial derivatives of $f$ are bounded and continuous.

$(P 2)$ there are $c_{0}, c_{1}>0$ such that $g(u, 0)<0$ for $u \geq c_{0}$ and $h(0, v)<0$ for $v \geq c_{1}$.

The following theorem is our main result on coexistence for the general competition model.

Theorem 4.1. Suppose

(A) $\lambda_{1}\left(-g\left(0, \theta_{h(0, \cdot)}\right)\right)<0, \lambda_{1}\left(-h\left(\theta_{g(\cdot, 0)}, 0\right)\right)<0$, where in general, $\lambda_{1}(q)$ is the smallest eigenvalue of $-\Delta+q$ with homogeneous boundary conditions, denoted simply by $\lambda_{1}$ when $q \equiv 0$, 
(B) (5) has a unique coexistence state $(u, v)$,

$(C)$ the Frechet derivative of $(5)$ at $(u, v)$ is invertible.

Then there is a neighborhood $V$ of $(g, h)$ in $\left[C_{B}^{1}\left(R^{2}\right)\right]^{2}$ such that if $(\bar{g}, \bar{h}) \in V$, then

(5) with $(\bar{g}, \bar{h})$ has a unique coexistence state.

Biologically, the first condition in Theorem 4.1 indicates that the rates of reproduction are relatively large. Similarly, the third condition, which requires the invertibility of the Frechet derivative, signifies that the rates of self-limitation are relatively larger than the rates of competition, a relationship that will be established in Lemma 4.2. When these conditions are fulfilled, the conclusion of our theorem asserts that small perturbations of the rates do not affect the existence and uniqueness of the positive steady state. That is, the two species implied can continue to coexist even if the factors determining the population densities vary slightly.

Now, at first glance, Theorem 4.1 may appear to be a consequence of the Implicit Function Theorem. However, the Implicit Function Theorem only guarantees local uniqueness. In contrast, our results in Theorem 4.1 guarantee global uniqueness. The techniques we will use in the proof of Theorem 4.1 include the Implicit Function Theorem and a priori estimates on solutions of (5).

Proof. Since the Frechet derivative of $(5)$ at $(u, v)$ is invertible, then, by the Implicit Function Theorem, there is a neighborhood $V$ of $(g, h)$ in $C_{B}^{1}$ and a neighborhood $W$ of $(u, v)$ in $\left[C_{0}^{2, \alpha}(\bar{\Omega})\right]^{2}$ such that for all $(\bar{g}, \bar{h}) \in V$, there is a unique positive solution $(\bar{u}, \bar{v}) \in W$ of (5). Thus, the local uniqueness of the solution is guaranteed.

To prove global uniqueness, suppose that the conclusion of Theorem 4.1 is false. Then there are sequences $\left(g_{n}, h_{n}, u_{n}, v_{n}\right),\left(g_{n}, h_{n}, \overline{u_{n}}, \overline{v_{n}}\right)$ in $V \times\left[C_{0}^{2, \alpha}(\bar{\Omega})\right]^{2}$ such that $\left(u_{n}, v_{n}\right)$ and $\left(\overline{u_{n}}, \overline{v_{n}}\right)$ are positive solutions of $(5)$ with $\left(g_{n}, h_{n}\right)$ and $\left(u_{n}, v_{n}\right) \neq$ $\left(\overline{u_{n}}, \overline{v_{n}}\right)$ and $\left(g_{n}, h_{n}\right) \rightarrow(g, h)$. By the Schauder's estimate in elliptic theory and the solution estimate in the proof of Theorem 2.3 , there are constants $k_{1}>0, k_{2}>0$ such that

$$
\begin{aligned}
& \left|u_{n}\right|_{2, \alpha} \leq k_{1} \sup _{x \in \bar{\Omega}}\left(u_{n}(x)\right) \leq k_{1} \sup _{x \in \bar{\Omega}}\left(\theta_{g_{n}(\cdot, 0)}(x)\right), \\
& \left|v_{n}\right|_{2, \alpha} \leq k_{2} \sup _{x \in \bar{\Omega}}\left(v_{n}(x)\right) \leq \sup _{x \in \bar{\Omega}}\left(\theta_{h_{n}(0, \cdot)}(x)\right),
\end{aligned}
$$

for all $n=1,2, \ldots$.

But, by the convergence of $\left\{g_{n}, h_{n}\right\}$ and the monotonicity of $\theta_{f}$, we conclude that $\left|u_{n}\right|_{2, \alpha}$ and $\left|v_{n}\right|_{2, \alpha}$ are uniformly bounded. Therefore, there are uniformly convergent subsequences of $\left\{u_{n}\right\}$ and $\left\{v_{n}\right\}$, which again will be denoted by $\left\{u_{n}\right\}$ and $\left\{v_{n}\right\}$.

Thus, let

$$
\begin{aligned}
& \left(u_{n}, v_{n}\right) \rightarrow(\hat{u}, \hat{v}), \\
& \left(\overline{u_{n}}, \overline{v_{n}}\right) \rightarrow(\bar{u}, \bar{v})
\end{aligned}
$$

Then $(\hat{u}, \hat{v}),(\bar{u}, \bar{v}) \in\left(C^{2, \alpha}\right)^{2}$ are also solutions of $(5)$ with $(g, h)$. We claim that $\hat{u}>0, \hat{v}>0, \bar{u}>0, \bar{v}>0$. To prove this assertion, it is sufficient to show that $\hat{u}$ and $\hat{v}$ are not identically zero because of the Maximum Principle.

Using proof by contradiction, we therefore suppose that either $\hat{u}$ or $\hat{v}$ is identically 
zero. Then by the Maximum Principle, one of the following cases should hold: (1) $\hat{u}$ is identically zero and $\hat{v}>0,(2) \hat{u}>0$ and $\hat{v}$ is identically zero, or (3) $\hat{u}$ is identically zero and $\hat{v}$ is identically zero.

Without loss of generality, assume that $\hat{u}$ is identically zero.

Now, let $\hat{u_{n}}=\frac{u_{n}}{\left\|u_{n}\right\|_{\infty}}, \hat{v_{n}}=v_{n}$ for all $n \in N$. Then

$$
\left\{\begin{array}{l}
\Delta \hat{u_{n}}+\hat{u_{n}} g_{n}\left(u_{n}, \hat{v_{n}}\right)=0 \\
\Delta \hat{v_{n}}+\hat{v_{n}} h_{n}\left(u_{n}, \hat{v_{n}}\right)=0
\end{array} \text { in } \Omega .\right.
$$

From the elliptic theory, $\hat{u_{n}} \rightarrow u_{0}$ and

$$
\left\{\begin{array}{l}
\Delta u_{0}+u_{0} g(0, \hat{v})=0 \\
\Delta \hat{v}+\hat{v} h(0, \hat{v})=0
\end{array} \text { in } \Omega,\right.
$$

by the continuity and uniform convergence.

Then, either (1) $\hat{v} \equiv 0$ or (2) $\hat{v}>0$. We must consider both cases:

(1) If $\hat{v} \equiv 0$, then $\lambda_{1}-g(0,0)=\lambda_{1}(-g(0,0))=0$, and so $g(0,0)=\lambda_{1}$, which contradicts our assumption.

(2) If $\hat{v}>0$,then $\hat{v}=\theta_{h(0, \cdot)}$ and so $\lambda_{1}\left(-g\left(0, \theta_{h(0, \cdot)}\right)\right)=\lambda_{1}(-g(0, \hat{v}))=0$, which is also a contradiction to our assumption.

Therefore, $\hat{u}$ and $\hat{v}$ are not identically zero. By the same procedure, $\bar{u}$ and $\bar{v}$ are also not identically zero. Then, by the Maximum Principle, $\hat{u}, \hat{v}, \bar{u}, \bar{v}>0$. Consequently, $(\hat{u}, \hat{v})$ and $(\bar{u}, \bar{v})$ are two coexistence states for $(5)$ with $(g, h)$. But, since the second condition of Theorem 4.1 states that the coexistence state with respect to $(g, h)$ is unique, then $(\hat{u}, \hat{v})=(\bar{u}, \bar{v})=(u, v)$. However, because $\left(u_{n}, v_{n}\right) \neq\left(\overline{u_{n}}, \overline{v_{n}}\right)$, we obtain a contradiction to the Implicit Function Theorem. Thus, Theorem 4.1 is established.

In biological terms, the proof of our theorem indicates that if one of two species living in the same domain becomes extinct, that is, if one species is excluded by the other, then the reproduction rates of both must be small. In other words, the region condition of reproduction rates $(A)$ is reasonable.

Now, the condition $(C)$ in Theorem 4.1 requiring the invertibility of the Frechet derivative is too artificial to have any direct biological implications. We therefore turn our attention to more applicable conditions that will guarantee the invertibility of the Frechet derivative. We then obtain the following relationship:

Lemma 4.2. Suppose $(u, v)$ is a positive solution to (5).

If $4 \inf \left(-g_{u}(u, v)\right) \inf \left(-h_{v}(u, v)\right) u v>\left[u \sup \left(g_{v}(u, v)\right)+v \sup \left(h_{u}(u, v)\right)\right]^{2}$, then the Frechet derivative of (5) at $(u, v)$ is invertible.

Proof. The Frechet derivative of $(5)$ at $(u, v)$ is

$$
A=\left(\begin{array}{ll}
-\Delta-g(u, v)-u g_{u}(u, v) & -u g_{v}(u, v) \\
-v h_{u}(u, v) & -\Delta-h(u, v)-v h_{v}(u, v)
\end{array}\right) .
$$

We need to show that $N(A)=\{0\}$ by the Fredholm Alternative, where $N(A)$ is the null space of $A$. If

$$
\left\{\begin{array}{l}
-\Delta \varphi+\left[-g(u, v)-u g_{u}(u, v)\right] \varphi-g_{v}(u, v) u \psi=0 \\
-\Delta \psi-h_{u}(u, v) v \varphi+\left[-h(u, v)-v h_{v}(u, v)\right] \psi=0
\end{array}\right.
$$


then

$$
\begin{aligned}
& \int_{\Omega}\left[|\nabla \varphi|^{2}+\left\{-g(u, v)-u g_{u}(u, v)\right\} \varphi^{2}-g_{v}(u, v) u \varphi \psi\right] d x=0 \\
& \int_{\Omega}\left[|\nabla \psi|^{2}-h_{u}(u, v) v \varphi \psi+\left\{-h(u, v)-v h_{v}(u, v)\right\} \psi^{2}\right]=0 .
\end{aligned}
$$

Since $\lambda_{1}(-g(u, v))=\lambda_{1}(-h(u, v))=0$, then

$$
\begin{aligned}
& \int_{\Omega}\left[|\nabla \varphi|^{2}-\left(g_{1}(u)-g_{2}(v)\right) \varphi^{2}\right] \geq 0, \\
& \int_{\Omega}\left[|\nabla \psi|^{2}-\left(h_{1}(v)-h_{2}(u)\right) \psi^{2}\right] \geq 0 .
\end{aligned}
$$

Hence,

$$
\begin{aligned}
& \int_{\Omega}\left(-u g_{u}(u, v) \varphi^{2}-g_{v}(u, v) u \varphi \psi\right) \leq 0 \\
& \int_{\Omega}\left(-h_{u}(u, v) v \varphi \psi-h_{v}(u, v) v \psi^{2}\right) \leq 0
\end{aligned}
$$

Thus, $\int_{\Omega}\left[-u g_{u}(u, v) \varphi^{2}+\left(-g_{v}(u, v) u-h_{u}(u, v) v\right) \varphi \psi-h_{v}(u, v) v \psi^{2}\right] \leq 0$.

We therefore find that if

$$
4 \inf \left(-g_{u}(u, v)\right) \inf \left(-h_{v}(u, v)\right) u v>\left[u \sup \left(g_{v}(u, v)\right)+v \sup \left(h_{u}(u, v)\right)\right]^{2}
$$

, then the integrand on the left side of the above inequality has positive definite form in $\Omega$, which means that $\varphi \equiv \psi \equiv 0$. Therefore, the Frechet derivative $A$ is invertible.

Combining Theorem 2.3, Theorem 4.1, and Lemma 4.2, we obtain the following corollary, which is the main result in this section. The importance of Corollary 4.3 is that it improves the results found by Kang, Oh, and Lee, as described in Theorem 2.3.

Corollary 4.3. Suppose

(A) $g\left(0, c_{1}\right)>\lambda_{1}, h\left(c_{0}, 0\right)>\lambda_{1}$, and

$(B)$

$$
\begin{aligned}
4 \inf \left(-g_{u}(u, v)\right) \inf \left(-h_{v}(u, v)\right) \geq & \frac{\theta_{g(\cdot, 0)}}{\theta_{h\left(c_{0}, \cdot\right)}}\left(\sup \left(g_{v}(u, v)\right)\right)^{2}+\frac{\theta_{h(0, \cdot)}}{\theta_{g\left(\cdot, c_{1}\right)}}\left(\sup \left(h_{u}(u, v)\right)\right)^{2} \\
& +2 \sup \left(g_{v}(u, v)\right) \sup \left(h_{u}(u, v)\right) .
\end{aligned}
$$

Then there is a neighborhood $V$ of $(g, h)$ in $\left(C_{B}^{1}\right)^{2}$ such that if $(\bar{g}, \bar{h}) \in V$, then (5) with $(\bar{g}, \bar{h})$ has a unique coexistence state.

Proof. Since $\theta_{g(\cdot, 0)}<c_{0}, \theta_{h(0, \cdot)}<c_{1}$, then by the monotonicity of $g, h$, and $\lambda_{1}$, we have

$$
\begin{aligned}
& \lambda_{1}\left(-g\left(0, \theta_{h(0, \cdot)}\right)\right) \leq \lambda_{1}\left(-g\left(0, c_{1}\right)\right)=\lambda_{1}-g\left(0, c_{1}\right)<0 \\
& \lambda_{1}\left(-h\left(\theta_{g(\cdot, 0)}, 0\right)\right) \leq \lambda_{1}\left(-h\left(c_{0}, 0\right)\right)=\lambda_{1}-h\left(c_{0}, 0\right)<0 .
\end{aligned}
$$

By Theorem 2.3, (5) has a unique coexistence state $(u, v)$. Furthermore, by the estimate of the solution in the proof of Theorem 2.3,

$$
\begin{aligned}
& 4 \inf \left(-g_{u}\right) \inf \left(-h_{v}\right) \\
> & \frac{\theta_{g(\cdot, 0)}}{\theta_{h\left(c_{0}, \cdot\right)}}\left(\sup \left(g_{v}(u, v)\right)\right)^{2}+\frac{\theta_{h(0, \cdot)}}{\theta_{g\left(\cdot, c_{1}\right)}}\left(\sup \left(h_{u}(u, v)\right)\right)^{2} \\
& +2 \sup \left(g_{v}(u, v)\right) \sup \left(h_{u}(u, v)\right) \\
\geq \quad & \frac{u}{v}\left[\sup \left(g_{v}(u, v)\right)\right]^{2}+\frac{v}{u}\left[\sup \left(h_{u}(u, v)\right)\right]^{2}+2 \sup \left(g_{v}(u, v)\right) \sup \left(h_{u}(u, v)\right) .
\end{aligned}
$$

Thus, we find that

$$
4 \inf \left(-g_{u}(u, v)\right) \inf \left(-h_{v}(u, v)\right) u v>\left[u \sup \left(g_{v}(u, v)\right)+v \sup \left(h_{u}(u, v)\right)\right]^{2} .
$$

By Lemma 4.2, this result implies that the Frechet derivative of $(5)$ at $(u, v)$ is invertible. Therefore, Corollary 4.3 follows from Theorem 4.1. 
In biological terms, the results obtained in Corollary 4.3 confirm that under certain conditions, two species who relax ecologically can continue to coexist at fixed rates. The requirements given in $(A)$ and $(B)$ simply state that each species must interact strongly with itself and weakly with the other species.

\section{5. perturbation of region}

Consider the model

$$
\left\{\begin{array}{c}
\Delta u+u g(u, v)=0 \\
\Delta v+v h(u, v)=0 \\
\left.u\right|_{\partial \Omega}=\left.v\right|_{\partial \Omega}=0 .
\end{array} \text { in } \Omega,\right.
$$

Here $\Omega$ is a bounded smooth domain in $R^{n}$ and $g, h \in C_{B}^{1}=\left\{f: R^{2} \rightarrow R \in\right.$ $C^{1} \mid f$ is strictly decreasing with respect to $u$ and $v$, $f$ and all of it's first order partial derivatives are bounded\}.

The following theorem is the main result.

TheOREM 5.1. Suppose

(A) $\Gamma$ is a closed, bounded, convex region in $C_{B}^{1}$ such that for all $(g, h) \in \Gamma$,

$$
\lambda_{1}\left(-g\left(0, \theta_{h(0, \cdot)}\right)\right)<0 \text { and } \lambda_{1}\left(-h\left(\theta_{g(\cdot, 0)}, 0\right)\right)<0 \text {, }
$$

(B) there exist $c_{0}>0$ and $c_{1}>0$ such that for all $(g, h) \in \Gamma, g(u, 0) \leq 0$ for $u \geq c_{0}$ and $h(0, v) \leq 0$ for $v \geq c_{1}$,

$(C)$ (6) has a unique positive solution for every $(g, h) \in \partial_{L} \Gamma$,

where $\partial_{L} \Gamma=\left\{\left(\lambda_{h}, h\right) \in \Gamma \mid\right.$ for any fixed $\left.h, \lambda_{h}=\inf \{\|g\| \mid(g, h) \in \Gamma\}\right\}$,

(D) for all $(g, h) \in \Gamma$, the Frèchet derivative of (6) at every positive solution to (6) is invertible.

Then for all $(g, h) \in \Gamma,(6)$ has a unique positive solution. Furthermore, there is an open set $W$ in $\left[C_{B}^{1}\right]^{2}$ such that $\Gamma \subseteq W$ and for every $(g, h) \in W,(6)$ has a unique positive solution.

Theorem 5.1 goes even further than Theorem 4.1 which states uniqueness in the whole region of $(g, h)$ whenever we have uniqueness on the left boundary and invertibility of the linearized operator at any particular solution inside the domain.

Proof. For each fixed $h$, consider $(g, h) \in \partial_{L} \Gamma$ and $(\bar{g}, h) \in \Gamma$. We need to show that for all $0 \leq t \leq 1$, (6) with $(1-t)(g, h)+t(\bar{g}, h)$ has a unique positive solution. Since $(6)$ with $(g, h)$ has a unique positive solution $(u, v)$ and the Frechet derivative of $(6)$ at $(u, v)$ is invertible, Theorem 4.1 implies that there is an open neighborhood $V$ of $(g, h)$ in $\left(C_{B}^{1}\right)^{2}$ such that if $\left(g_{0} . h_{0}\right) \in V$, then $(6)$ with $\left(g_{0}, h_{0}\right)$ has a unique positive solution. Let $\lambda_{s}=\sup \{0 \leq \lambda \leq 1 \mid(6)$ with $(1-t)(g, h)+$ $t(\bar{g}, h)$ has a unique coexistence state for $0 \leq t \lambda$. $\}$. We need to show that $\lambda_{s}=1$. Suppose $\lambda_{s}<1$. From the definition of $\lambda_{s}$, there is a sequence $\left\{\lambda_{n}\right\}$ such that $\lambda_{n} \rightarrow \lambda_{s}^{-}$and there is a sequence $\left(u_{n}, v_{n}\right)$ of the unique positive solution of (6) with $\left(1-\lambda_{n}\right)(g, h)+\lambda_{n}(\bar{g}, h)$. Then by the elliptic theory, there is $\left(u_{0}, v_{0}\right)$ such that $\left(u_{n}, v_{n}\right)$ converges to $\left(u_{0}, v_{0}\right)$ uniformly and $\left(u_{0}, v_{0}\right)$ is a solution of $(6)$ with $\left(1-\lambda_{s}\right)(g, h)+\lambda_{s}(\bar{g}, h)$. We claim that both $u_{0}$ and $v_{0}$ are not identically zero. Suppose this is false. Then by the Maximum Principles, either one of the following three cases holds.

(1) $u_{0}$ is identically zero and $v_{0}>0$.

(2) $u_{0}>0$ and $v_{0}$ is identically zero. 
(3) Both $u_{0}$ and $v_{0}$ are identically zero.

(1) Suppose $u_{0}$ is identically zero.

Let $\tilde{u_{n}}=\frac{u_{n}}{\left\|u_{n}\right\|_{\infty}}$ for all $n \in N$. Then

$$
\begin{aligned}
& \Delta \tilde{u_{n}}+\tilde{u_{n}}\left(\left(1-\lambda_{n}\right) g\left(u_{n}, v_{n}\right)+\lambda_{n} \bar{g}\left(u_{n}, v_{n}\right)\right)=0, \\
& \Delta v_{n}+v_{n} h\left(u_{n}, v_{n}\right)=0,
\end{aligned}
$$

and $\tilde{u_{n}} \rightarrow \tilde{u}$ uniformly in $\Omega$ by elliptic theory, and

$$
\begin{aligned}
& \Delta \tilde{u}+\tilde{u}\left(\left(1-\lambda_{s}\right) g\left(0, v_{0}\right)+\lambda_{s} \bar{g}\left(0, v_{0}\right)\right)=0, \\
& \Delta v_{0}+v_{0} h\left(0, v_{0}\right)=0,
\end{aligned}
$$

It implies that $\lambda_{1}\left(-\left(\left(1-\lambda_{s}\right) g\left(0, v_{0}\right)+\lambda_{s} \bar{g}\left(0, v_{0}\right)\right)\right)=0$. If $v_{0}>0$, then $\lambda_{1}(-((1-$ $\left.\left.\left.\lambda_{s}\right) g\left(0, \theta_{h(0, \cdot)}\right)+\lambda_{s} \bar{g}\left(0, \theta_{h(0, \cdot)}\right)\right)\right)=0$, which is a contradiction to our assumption since $\left(\left(1-\lambda_{s}\right) g+\lambda_{s} \bar{g}, h\right) \in \Gamma$. If $v_{0}$ is identically zero, then by the monotonicity, $0=\lambda_{1}\left(-\left(\left(\left(1-\lambda_{s}\right) g+\lambda_{s} \bar{g}\right)(0,0)\right)\right)<\lambda_{1}\left(-\left(\left(\left(1-\lambda_{s}\right) g+\lambda_{s} \bar{g}\right)\left(0, \theta_{h(0, \cdot)}\right)\right)\right.$, which is also a contradiction to the same assumption.

(2) Suppose $u_{0}>0$ and $v_{0}$ is identically zero.

Let $\tilde{v}_{n}=\frac{v_{n}}{\left\|v_{n}\right\|_{\infty}}$ for all $n \in N$. Then

$$
\begin{aligned}
& \Delta u_{n}+u_{n}\left(\left(1-\lambda_{n}\right) g\left(u_{n}, v_{n}\right)+\lambda_{n} \bar{g}\left(u_{n}, v_{n}\right)\right)=0, \\
& \Delta \tilde{v_{n}}+\tilde{v_{n}} h\left(u_{n}, v_{n}\right)=0,
\end{aligned}
$$

and $\tilde{v_{n}} \rightarrow \tilde{v}$ uniformly in $\Omega$ by elliptic theory, and

$$
\begin{aligned}
& \Delta u_{0}+u_{0}\left(\left(1-\lambda_{s}\right) g\left(u_{0}, 0\right)+\lambda_{s} \bar{g}\left(u_{0}, 0\right)\right)=0, \\
& \Delta \tilde{v}+\tilde{v} h\left(u_{0}, 0\right)=0
\end{aligned}
$$

It implies that $u_{0}=\theta_{\left(\left(1-\lambda_{s}\right) g+\lambda_{s} \bar{g}\right)(\cdot, 0)}$ and $\lambda_{1}\left(-h\left(u_{0}, 0\right)\right)=0$.

Hence, $\lambda_{1}\left(-h\left(\theta_{\left(\left(1-\lambda_{s}\right) g+\lambda_{s} \bar{g}\right)(\cdot, 0)}, 0\right)\right)=0$, which is a contradiction to our assumption since $\left(\left(1-\lambda_{s}\right) g+\lambda_{s} \bar{g}, h\right) \in \Gamma$.

Thus $u_{0}, v_{0}$ are not identically zero. We claim that (6) has a unique coexistence state with $\left(1-\lambda_{s}\right)(g, h)+\lambda_{s}(\bar{g}, h)$. In fact, if not, assume that $\left(\overline{u_{0}}, \overline{v_{0}}\right) \neq\left(u_{0}, v_{0}\right)$ is another coexistence state. By the Implicit Function Theorem, there exists $0<\tilde{a}<\lambda_{s}$ and very close to $\lambda_{s},(6)$ with $(1-\tilde{a})(g, h)+\tilde{a}(\bar{g}, h)$ has a coexistence state very close to $\left(\overline{u_{0}}, \overline{v_{0}}\right)$ which means that $(6)$ with $(1-\tilde{a})(g, h)+\tilde{a}(\bar{g}, h)$ has more than one coexistence state. This is a contradiction to the definition of $\lambda_{s}$. But, since (6) with $\left(1-\lambda_{s}\right)(g, h)+\lambda_{s}(\bar{g}, h)$ has a unique coexistence state and the Frechet derivative is invertible, Theorem 4.1 concluded that $\lambda_{s}$ can not be as defined. Therefore, for each $(g, h) \in \Gamma,(6)$ with $(g, h)$ has a unique coexistence state $(u, v)$. Furthermore, by the assumption, for each $(g, h) \in \Gamma$, the Frechet derivative of $(6)$ with $(g, h)$ at the unique solution $(u, v)$ is invertible. Hence, Theorem 4.1 concluded that there is an open neighborhood $V_{(g, h)}$ of $(g, h)$ in $\left(C_{B}^{1}\right)^{2}$ such that if $(\bar{g}, \bar{h}) \in V_{(g, h)}$, then (6) with $(\bar{g}, \bar{h})$ has a unique coexistence state. Let $W=\bigcup_{(g, h) \in \Gamma} V_{(g, h)}$. Then $W$ is an open set in $\left(C_{B}^{1}\right)^{2}$ such that $\Gamma \subseteq W$ and for each $(\bar{g}, \bar{h}) \in W,(6)$ with $(\bar{g}, \bar{h})$ has a unique coexistence state.

Apparently, Theorem 5.1 generalizes Theorem 4.1 and consequently, we have the following result which is actually the main conclusion in this section. 
Corollary 5.2. Suppose

(A) $\Gamma$ is a closed, bounded, convex region in $\left[C_{B}^{1}\right]^{2}$,

$(B)$ there exist $c_{0}>0$ and $c_{1}>0$ such that for all $(g, h) \in \Gamma, g\left(0, c_{1}\right)>\lambda_{1}, h\left(c_{0}, 0\right)>$

$\lambda_{1}, g(u, 0) \leq 0$ for $u \geq c_{0}$ and $h(0, v) \leq 0$ for $v \geq c_{1}$,

(C) for all $(g, h) \in \Gamma$,

$$
\begin{aligned}
& 4 \inf \left(-g_{x}\right) \inf \left(-h_{y}\right) \\
\geq \quad & \frac{\theta_{g(\cdot, 0)}}{\theta_{h\left(c_{0}, \cdot\right)}}\left[\sup \left(g_{y}\right)\right]^{2}+\frac{\theta_{h(0, \cdot)}}{\theta_{g\left(\cdot, c_{1}\right)}}\left[\sup \left(h_{x}\right)\right]^{2}+\left[\sup \left(g_{y}\right)\right]\left[\sup \left(h_{x}\right)\right] .
\end{aligned}
$$

Then there is an open set $W$ in $\left[C_{B}^{1}\right]^{2}$ such that $\Gamma \subseteq W$ and for every $(g, h) \in W$, (6) with $(g, h)$ has a unique positive solution.

Proof. From $\theta_{g(\cdot, 0)} \leq c_{0}, \theta_{h(0, \cdot)} \leq c_{1}$ and the monotonicity, we have

$$
\begin{aligned}
& \lambda_{1}\left(-g\left(0, \theta_{h(0, \cdot)}\right)\right)<\lambda_{1}\left(-g\left(0, c_{1}\right)\right)=\lambda_{1}-g\left(0, c_{1}\right)<0, \\
& \lambda_{1}\left(-h\left(\theta_{g(\cdot, 0)}, 0\right)\right)<\lambda_{1}\left(-h\left(c_{0}, 0\right)\right)=\lambda_{1}-h\left(c_{0}, 0\right)<0
\end{aligned}
$$

for all $(g, h) \in \Gamma$.

By the condition $(C)$ and the Theorem 2.3, (6) has a unique positive solution for all $(g, h) \in \partial_{L} \Gamma$.

Furthermore, by the estimate of the solution in the proof of Theorem 2.3, if $(u, v)$ is a positive solution of $(6)$ with $(g, h) \in \Gamma$, then

$$
4 \inf \left(-g_{x}\right) \inf \left(-h_{y}\right)>\left[u \sup \left(g_{y}\right)+v \sup \left(h_{x}\right)\right]^{2} .
$$

Hence, by the Lemma 4.2 , if $(u, v)$ is a positive solution of $(6)$ for $(g, h) \in \Gamma$, then the Frechet derivative of $(6)$ at $(u, v)$ is invertible. Therefore, the Theorem follows from the Theorem 5.1.

\section{Conclusions}

In this paper, our investigation of the effects of perturbations on the general competition model resulted in the development and proof of Theorem 4.1, Lemma 4.2 , and Corollary 4.3, as detailed above. The three together assert that given the existence of a unique solution $(u, v)$ to the system (5), perturbations of the functions $g(u, v)$ and $h(u, v)$, within a specified neighborhood, will maintain the existence and uniqueness of the positive steady state. Indeed, our results specifically outline conditions sufficient to maintain the positive, steady state solution when the general competition model is perturbed within some region.

Applying this mathematical result to real world situations, our results establish that two species residing in the same environment can vary their interactions, within certain bounds, and continue to survive together indefinitely at unique densities. The conditions necessary for coexistence, as described in the theorem, simply require that members of each species interact strongly with themselves and weakly with members of the other species.

The research presented in this paper has a number of strengths, which confirm both the validity and the applicability of the project. First, the mathematical conditions required in Corollary 4.3 are identical to those required in Theorem 2.3 developed by Kang, Oh, and Lee. However, they used these conditions to prove the 
existence and uniqueness of the positive steady state solution for the general competition model. In contrast, our theorem employs the same conditions to establish that the existence and uniqueness of this solution is maintained when the model is perturbed within some neighborhood. Thus, our findings extend and improve established mathematical theory.

Secondly, perturbations of the general model render its implications more applicable both mathematically and biologically. Because our theorem extends the steady state to any function $(\bar{g}, \bar{h})$ within some neighborhood of $(g, h)$, results for the general model pertain to a far wider variety of functions. Biologically, perturbations extend the model's description to species affected by factors that vary slightly yet erratically. Thus, the description of competitive interactions given by the model becomes a closer approximation of real-world population dynamics.

While our research therefore represents a progression in the field, the results obtained have two important limitations. First, Theorem 4.1, Lemma 4.2, and Corollary 4.3 establish that a region of perturbation exists within which the coexistence state is maintained for the general competition model. However, the exact extent of that region remains unknown. Second, our model describes the interactions of only two species who reside in the same environment. The conditions required for the coexistence of more than two species have not been investigated.

Therefore, the results presented in this paper may serve as a platform for research of the two questions given above. Mathematicians should now attempt to establish the exact extent of the perturbation region in which coexistence is maintained for the general model. Such information would prove very useful not only mathematically but also biologically. Specifically, knowledge of the extent of the region would imply exactly how far two species can relax and yet continue to coexist. Second, future research should also focus on systems involving more than two populations. Mathematically, analysis of competition models for $n$ populations would expand the community's understanding of the behavior of functions and extend established theory in the field. Biologically, the investigation of models for $n$ species would increase knowledge on the nature of interactions between any number of species within the same environment. Thus, the results achieved through our research will

enable both fields to continue the development of theory on competitive interaction of populations.

\section{References}

[1] S.W. Ali and C. Cosner, On the uniqueness of the positive steady state for Lotka-Volterra Models with diffusion, Journal of Mathematical Analysis and Application, 168(1992), 329341.

[2] R.S. Cantrell and C. Cosner, On the steady-state problem for the Volterra-Lotka competition model with diffusion, Houston Journal of Mathematics, 13(1987), 337-352.

[3] R. S. Cantrell and C. Cosner, On the uniqueness and stability of positive solutions in the Volterra-Lotka competition model with diffusion, Houston J. Math. 15(1989) 341-361.

[4] C. Cosner and A. C. Lazer, Stable coexistence states in the Volterra-Lotka competition model with diffusion, Siam J. Appl. Math., 44(1984), 1112-1132.

[5] D. Dunninger, Lecture note for applied analysis in Michigan State University.

[6] J. L.-Gomez and R. Pardo, Existence and uniqueness for some competition models with diffusion, C.R. Acad. Sci. Paris, Ser.I math. 313(1991), 933-938. 
[7] C. Gui and Y. Lou, Uniqueness and nonuniqueness of coexistence states in the Lotka-Volterra competition model, Comm Pure and Appl. Math., XVL2, No.12(1994), 1571-1594.

[8] J. Kang and Y. Oh, A sufficient condition for the uniqueness of positive steady state to a reaction diffusion system, Journal of Korean Mathematical Society, Vol.39, No.39(2002), 377-385.

[9] J. Kang and Y. Oh, Uniqueness of Coexistence State of General Competition Model for Several Competing Species, Kyungpook Mathematical Journal, Vol.42, No.2(2002), 391-398.

[10] J. Kang, Y. Oh, and J. Lee, The existence, nonexistence and uniqueness of global positive coexistence of a nonlinear elliptic biological interacting model, Kangweon-Kyungki Math. Jour.12(2004), No.1, pp.77-90.

[11] L. Li and R. Logan, Positive solutions to general elliptic competition models, Differential and Integral Equations, 4(1991), 817-834.

[12] A. Leung, Equilibria and stabilities for competing-species, reaction-diffusion equations with Dirichlet boundary data, J. Math. Anal. Appl., 73(1980), 204-218.

Department of Mathematics, Andrews University, Berrien Springs, Mi 49104

Department of Mathematics, Andrews University, Berrien Springs, Mi 49104

E-mail address: kang@andrews.edu

Department of Mathematics, Michigan State University, East Lansing, Mi 48823 